\title{
Towards Mobile Augmented Reality for On-Patient Visualization of Medical Images
}

\author{
L. Maier-Hein ${ }^{1}$, A. M. Franz ${ }^{1}$, M. Fangerau ${ }^{1}$, M. Schmidt ${ }^{2}$, A. Seitel ${ }^{1}$, \\ S. Mersmann ${ }^{1}$, T. Kilgus ${ }^{1}$, A. Groch ${ }^{1}$, K. Yung ${ }^{1}$, T. R. dos Santos ${ }^{1}$, \\ H.-P. Meinzer ${ }^{1}$ \\ ${ }^{1}$ German Cancer Research Center, Div. of Medical and Biological Informatics \\ ${ }^{2}$ Heidelberg Collaboratory for Image Processing, University of Heidelberg \\ 1.maier-hein@dkfz-heidelberg.de
}

\begin{abstract}
Despite considerable technical and algorithmic developments related to the fields of medical image acquisition and processing in the past decade, the devices used for visualization of medical images have undergone rather minor changes. As anatomical information is typically shown on monitors provided by a radiological work station, the physician has to mentally transfer internal structures shown on the screen to the patient. In this work, we present a new approach to on-patient visualization of 3D medical images, which combines the concept of augmented reality (AR) with an intuitive interaction scheme. The method requires mounting a Time-of-Flight (ToF) camera to a portable display (e.g., a tablet PC). During the visualization process, the pose of the camera and thus the viewing direction of the user is continuously determined with a surface matching algorithm. By moving the device along the body of the patient, the physician gets the impression of being able to look directly into the human body. The concept can be used for intervention planning, anatomy teaching and various other applications that require intuitive visualization of $3 \mathrm{D}$ data.
\end{abstract}

\section{Introduction}

Visualization of medical images for disease diagnosis, surgical planning or as orientation means during interventional therapy is an integral part of today's health care. As only few medical imaging modalities provide real-time images of the patient's anatomy, a common procedure involves acquiring static 3D images, e.g. with a computed tomography $(\mathrm{CT})$ or magnetic resonance imaging (MRI) scanner, and visualizing the data on a regular monitor provided by a radiological work station. In consequence, it is the task of the physician to transfer these images mentally to the patient. Furthermore, navigation in the 3D data set using the provided standard input devices may not be intuitive and thus requires experience with the system.

In related work, some promising methods have been proposed for improving visualization during interventional therapy via augmented reality (AR), e.g. by applying head-mounted displays [1] or intra-operative projector systems [2]. 
In general, however, these methods require relatively bulky equipment such as optical tracking systems and are thus not appropriate or too costly to be used in the context of diagnosis, teaching, or surgical planning. To address this issue, we present a new approach to on-patient visualization of anatomical data that provides an augmented reality (AR) view of the medical data as well as an intuitive interaction scheme without requiring expensive or bulky equipment.

\section{Materials and Methods}

\subsection{Visualization Concept}

The proposed visualization concept is based on a portable device such as a tablet PC or an iPad with a mounted Time-of-Flight (ToF) camera as shown in Fig. 1. The ToF camera is able to generate dense range images and corresponding grayscale intensity images from a given scene in real-time [3]. Optionally, a color camera calibrated with the ToF camera can be attached to the device to provide high-resolution color images of the scene.

To enable on-patient visualization of a given static 3D data set, the images stored in the Picture Archiving and Communications System (PACS) of the clinic are transferred to the portable viewer (e.g., via a wireless connection). Next, the skin as well as additional structures of interest are segmented (semi-) automatically with the medical image processing software provided by the device. Note in this context that the computations can optionally be performed remotely on an external server. The physician may then move the portable monitor freely along the body of the patient with the mounted ToF camera pointing in the viewing direction of the user as shown in Fig. 1. During this procedure, the pose of the ToF camera is continuously estimated by registering the ToF data with the surface extracted from the static image. In consequence, the viewing direction of the physician relative to the patient is known, and the virtual camera showing the 3D data may be set accordingly. By looking onto the screen, which provides a view on the internal anatomical structures using e.g. volume rendering or surface rendering techniques, the physician obtains a kind of "x-ray vision" into the patient. Furthermore, navigation through the 3D data set becomes more intuitive because it is performed directly at the object of interest.

Given the hardware, which is already commercially available, implementation of the concept thus requires real-time capable algorithms for (1) preprocessing of the camera data, (2) camera pose estimation, and (3) visualization.

\subsection{Feasibility Study}

To investigate the feasibility of the proposed visualization concept, we implemented a prototype based on the CamCube 2.0 (PMD Technologies, Siegen, Germany) and the open-source software framework The Medical Imaging Interaction Toolkit (MITK) (www.mitk.org) using the following methods to process each image: 


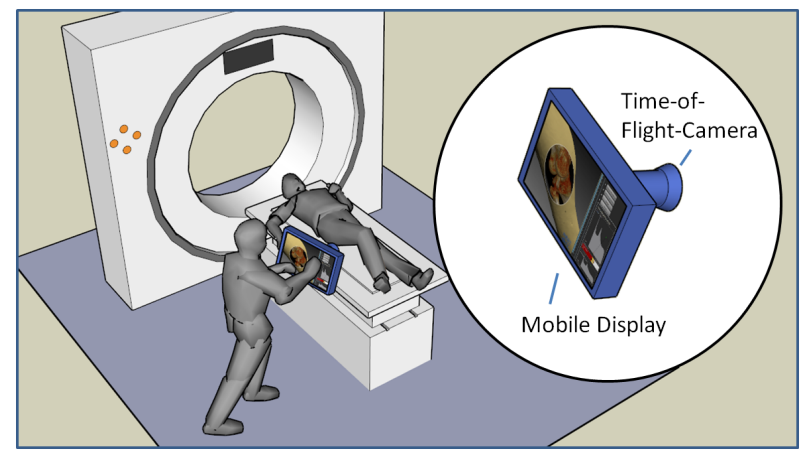

Fig. 1. Concept for on-patient visualization: the pose of a portable device relative to the patient is continuously estimated by registering time-of-flight range images to static volume data.

- Preprocessing: After distortion correction based on a standard calibration procedure for the intrinsic camera parameters, the range image is denoised using a variant of the bilateral filter that takes into account the intensity and distance measured in the individual pixels [4]. Next, a threshold filter is applied to the distance image to segment those parts of the image that correspond to the patient surface. Finally, the range image is converted into Cartesian coordinates, and a Delaunay based triangulation method is applied to generate a surface from the resulting point cloud.

- Camera pose estimation: In the current implementation, the pose of the camera is continuously computed by matching the surfaces generated from the ToF range images to the patient surface extracted from the static data set. Initially, i.e., upon start of the visualization process, a two-stage registration process is performed to get an alignment: (1) First, both meshes are segmented into regions sharing similar curvature properties, and a graph representation is generated, where each node represents a region and each edge connects neighboring regions. Next, a graph matching procedure [5] is applied to find a rough alignment of the surfaces. (2) Based on this alignment, an anisotropic variant of the iterative closest point (ICP) algorithm [6] is applied for the purpose of fine surface registration. The latter accounts for the high noise along the viewing direction of the camera. After convergence, the current transformation is used to get an initial alignment for the next image frame, i.e., step (1) has to be performed only once at the beginning of the procedure. Note in this context, that the registration problems arising from symmetric body parts are potentially eliminated by applying a registration procedure that converges only to the nearest local optimum with respect to a given cost function. Ambiguities can thus be compensated for, given that the alignment for the previous image frame was correct. This aspect should be accounted for upon initialization of the visualization process, i.e., by maximizing the area of the surface used for the initial alignment. 
- Visualization: In the current implementation, the physician may choose one of four visualization modes: Volume rendering, surface rendering, virtual $x$ ray view, and slice view, as shown in Fig. 2. Similar as in $[1,7]$, internal structures are presented in relation to the skin surface by virtually cutting a sphere-shaped hole into the skin as shown in Fig. 2. To avoid manipulation of the skin surface mesh, the cutting is realized during the rendering process using the concept of shading.

In a related contribution, we presented an evaluation framework which allows for assessing the performance of ToF registration methods by providing physically realistic ToF range data generated from a virtual scence [6]. In this work, the framework was applied to perform first experiments on the feasibility of continuous surface registration based on the anisotropic ICP: A sequence of five ToF images was simulated from the CT image of a human face, and the proposed registration method was applied to continuously register the simulated ToF range data with the static 3D data set starting with a small known misalignment. For each image frame (except for the first one), the estimated camera pose of the previous frame was used to initialize the fine registration algorithm. After convergence, the target registration error (TRE) for a set of virtual targets distributed in the brain of the patient was determined to quantify registration accuracy.

\section{Results}

A prototype for the visualization concept has successfully been implemented with the following results: (i) preprocessing and surface generation is performed robustly at 10 frames/sec on a standard PC; (ii) camera pose estimation based on surface registration with the anisotropic ICP was successful in our experiments, yielding a mean root-mean-square (RMS) TRE of $0.3 \pm 0.1 \mathrm{~mm}$ (final value: $0.2 \mathrm{~mm}$ ) compared to $2.1 \pm 0.5 \mathrm{~mm}$ (final value: $2.3 \mathrm{~mm}$ ) with the standard ICP; (iii) visualization using the different modes represented in Fig. 2 works in real-time.

\section{Discussion}

Compared to other visualization concepts introduced in the literature, the proposed method has several major advantages: It (i) is markerless, (ii) involves no bulky equipment, (iii) provides an intuitive mechanism for navigating through a 3D data set, and (iv) poses no restrictions on image acquisition (such as using a tracked imaging modality, attaching markers, etc.). A seamless integration into the clinical workflow, however, requires addressing several remaining issues. Firstly, improvement of the ToF surfaces by calibration of systematic distance errors is necessary to ensure robust registration results in practice. Secondly, a real-time implementation of the fine surface registration method based on 


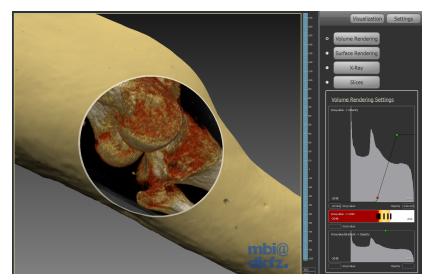

(a) volume rendering

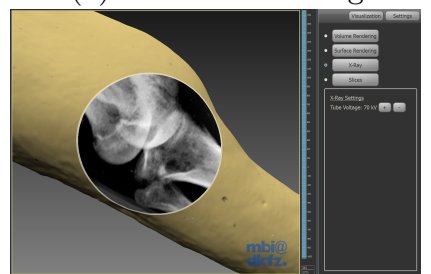

(c) x-ray view

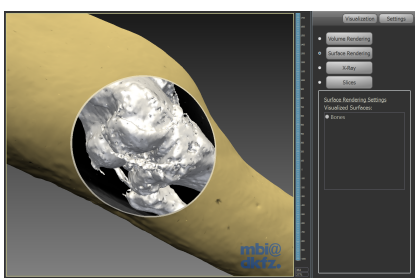

(b) surface rendering

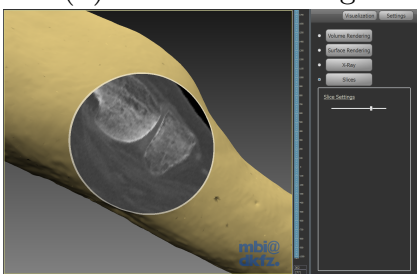

(d) slice view

Fig. 2. Visualization options. The $x$-ray view shows a simulated $\mathrm{x}$-ray from the viewing direction of the camera. In the slice view, the user may navigate through reconstructed image planes whose normals point in the viewing direction of the user.

optimizing and parallelizing the weighted nearest neighbor computation is necessary. In the long run, the registration method should further be able to cope with varying angles between joints as well as with changing clothing. Finally, the accuracy, run-time, and robustness of the method must be extensively evaluated with simulated and real data. The proposed concept could then become a valuable tool for $\mathrm{AR}$ visualization of $3 \mathrm{D}$ images in the context of port planning, anatomy teaching, and many other fields.

\section{References}

1. Navab N, et al. Action- and workflow-driven augmented reality for computer-aided medical procedures. IEEE Comput Graph Appl. 2007;27(5):10-4.

2. Sugimoto M, et al. Image overlay navigation by markerless surface registration in gastrointestinal, hepatobiliary and pancreatic surgery. J Hepatobiliary Pancreat Sci. 2010;17(5):629-36.

3. Kolb A, et al. Time-of-Flight Sensors in Computer Graphics. In: Proc Eurographics; 2009. p. 119-34.

4. Seitel A, et al. Adaptive bilateral filter for image denoising and its application to in-vitro Time-of-Flight data. Proc SPIE. 2011;(in press).

5. dos Santos TR, et al. Correspondences search for surface-based intra-operative registration. Lect Notes Computer Sci. 2010;6362:660-7.

6. Maier-Hein L, et al. Accounting for anisotropic noise in fine registration of Timeof-Flight range data with high-resolution surface data. Lect Notes Computer Sci. 2010;6361:251-8.

7. Tietjen C, et al. GPU-basierte Smart Visibility Techniken für die Planung von Tumor-Operationen. Proc BVM. 2009; p. 272-276. 